\title{
Incidence, Survival, and Treatment of Localized and Metastatic Neuroblastoma in Germany 1979-2015
}

\author{
Frank Berthold ${ }^{1}$ (D) Claudia Spix $^{2} \cdot$ Peter Kaatsch $^{2} \cdot$ Fritz Lampert $^{3}$
}

Published online: 7 August 2017

(C) The Author(s) 2017. This article is an open access publication

\begin{abstract}
Background A comprehensive clinical long-term survey over the complete spectrum of neuroblatoma disease is lacking in the literature.

Objective Our objective was to describe the incidence, risk profiles, therapies, and outcomes for the total cohort of German patients with neuroblastoma including all clinical stages and risk groups.

Methods Epidemiological, clinical, and outcome data of neuroblastoma patients who participated in one of the six consecutive national trials between 1979 and 2015 were analyzed retrospectively.

Results Of all German neuroblastoma patients known to the national childhood cancer registry, ninety seven percent enrolled in one of the trials. The absolute neuroblastoma rate has increased slightly, whereas the median age at diagnosis has decreased. Except for the screening period (1995-2000), the risk factors lactate dehydrogenase (LDH), ferritin, chromosome 1p, and the $M Y C N$ oncogene have remained largely constant, with the exception of an increase in $M Y C N$
\end{abstract}

Electronic supplementary material The online version of this article (doi:10.1007/s40272-017-0251-3) contains supplementary material, which is available to authorized users.

Frank Berthold

frank.berthold@uk-koeln.de

1 Department of Pediatric Oncology and Hematology, Children's Hospital, University of Cologne, Kerpener Strasse 62, 50924 Cologne, Germany

2 Institute of Medical Biostatistics, Epidemiology and Informatics, University Medical Center Mainz, Mainz, Germany

3 Department of Pediatric Oncology and Hematology, Children's Hospital, University of Giessen, Giessen, Germany amplification at stage 4 for those aged $\geq 18$ months between trials NB97 (27\%) and NB2004 (35\%). The 10-year overall survival increased in patients with stage 1-3 neuroblastoma from 83 to $91 \%$, for stage 4 S from 80 to $85 \%$, and for stage 4 aged $\geq 18$ months from 2 to $38 \%$. The fraction of patients in stages 1-3 who never received chemotherapy (neither for frontline nor at recurrence) increased from 35 to $60 \%$. The proportion of macroscopically complete surgical resections of the primary tumor decreased for the total population as well as for patients with stage 4 aged $\geq 18$ months. The impact of chemotherapy response on the outcome was trial dependent. The overall proportion of toxic death during the time of the protocol therapy was $6 \%$ for stage 4 patients aged $\geq 18$ months and $2 \%$ for low-/intermediate-risk patients. The most frequently reported late sequelae in stage 4 patients aged $\geq 18$ months were renal dysfunctions, hypothyroidism, major hearing impairment, and second malignancies.

Conclusion The body of data for incidences, risk profiles, and survival rates from this survey of more than 37 years provides a useful perspective for future studies on neuroblastoma sub-cohorts.

\section{Key Points}

The incidence of neuroblastoma increased slightly in Germany between 1986 and 2015.

Total event-free and overall survival increased significantly, although the levels achieved in the high-risk group are still unsatisfactory.

Improved understanding of risk groups has led to reduced treatment intensity in the low-risk group and to increased intensity in the high-risk group. 


\section{Introduction}

Neuroblastoma is a childhood cancer with remarkably divergent courses. Spontaneous regressions, chemotherapy-induced or spontaneous maturation, and highly malignant tumor progressions are nowadays seen in a predictable number of patients. Recent years have seen improved understanding of the molecular characteristics of neuroblastomas and their association with clinical outcomes $[1,2]$. This has resulted in a complex system with many subcategories [3, 4]. However, a comprehensive overview of a well-defined national cohort for the complete spectrum of the disease is lacking.

Therefore, the aim of this survey was to describe the incidence, risk profiles, therapies, and outcomes for the total cohort of German patients with neuroblastomas with all clinical stages and risk groups. The data from this research may provide a perspective for future studies that are conducted mainly in neuroblastoma sub-cohorts.

\section{Patients and Methods}

\subsection{Cohort of Analyzed Patients}

Patients with newly diagnosed neuroblastomas were reported primarily to the central German Childhood Cancer Registry at the University of Mainz. If the "wish to participate in trial" box was checked by the parents or the patient, the trial office received this information and then interacted directly with the treating institutions (which included informed consent, randomization, and reporting).

\subsubsection{Epidemiological Cohort}

Neuroblastoma incidence rates were calculated for periods between 1986 and 2015 on the basis of 3811 cases from the German Childhood Registry and yearly annual German public population numbers for children. Patients who had indicated consent to participate in the trials but lived outside of Germany were excluded. The number of cases before 1986 was not complete enough to allow epidemiological analysis.

\subsubsection{Trial Cohorts}

A total of 4459 consecutive patients were registered for the national trials NB79, NB82, NB85, NB90, NB97, and NB2004 of the German Society of Pediatric Oncology and Hematology (GPOH). The data were retrospectively analyzed. The patients represent $96.6 \%$ of all patients known to the central German Childhood Cancer Registry at the
University of Mainz [5]. The time period for recruitment spanned from 1 July 1979 to 31 December 2015. The data lock for follow-up was 30 September 2016. To cover the full spectrum of the disease, none of the registered patients was excluded, even when the recommended treatment elements could not be applied. All protocols were approved by the ethics committees of the Universities of Gießen (NB79, NB82, NB85) or Cologne (NB90, NB97, NB2004) as leading institutions, and approval was confirmed by the local ethics committees. Up to 85 institutions recruited patients and reported the relevant data to the trial office. Informed consent was obtained from the participants at the local treatment site and was a prerequisite to register in the trials.

\subsection{Diagnostic Criteria}

The diagnosis of neuroblastoma was established according to international criteria [6]. Diagnostic meta- ${ }^{123}$ iodine benzylguanidine (mIBG) scintigraphy and central bone marrow review were introduced into the regular diagnostic work-up in 1985. The type of radiological imaging techniques was left to the discretion of the local physicians of the participating hospitals. Bone marrow cytology remained the standard for the detection of tumor cell infiltration in the bone marrow throughout the data collection periods. Immunocytochemistry, using anti-GD2 antibody staining, was introduced in 1987 as a supplementary tool. Bone metastasis was defined as radiological evidence of bone lesions by X-ray or computed tomography (CT) in trials NB79-NB85, and by distinct and superimposable skeletal scintigraphic uptake of ${ }^{99} \mathrm{mTechnetium}$ and ${ }^{123} \mathrm{I}$ mIBG in trials NB90-NB97. In trial NB2004, skeletal investigations were performed via mIBG scintigraphy only. Difficult discrimination between bone marrow and bone involvement by mIBG scintigraphy led to a new radiodiagnostic definition of "osteomedullary metastasis," which comprises bone marrow and bone. Bone marrow cytology was not used to define "osteomedullary metastasis." Intracranial metastasis comprised metastases to the brain or the meninges, detection of tumor cells in the cerebrospinal fluid, and significant extension of a tumor lesion from the skull into the brain. Intracranial metastasis therefore is a wider definition than intracerebral metastasis.

\subsection{Risk factors and Risk Groups}

Serum lactate dehydrogenase (LDH, since 1979) and serum ferritin (since 1982) were investigated at the local site using age-related reference values for the categorization as "abnormal" versus "normal" [7]. MYCN amplification ( $\geq 5$ 
times of the normal reference) was analyzed twice in two different laboratories using two of the three available techniques (Southern blot, polymerase chain reaction, fluorescence in situ hybridization) since 1990 [8]. Chromosome $1 \mathrm{p}$ status (deletion, imbalance, normal) was also determined twice by fluorescence in situ hybridization in two different laboratories [9].

Risk group definitions of trials NB79, NB82, and NB85 were based only on the stage (stages $1-3$ vs. $4 \mathrm{~S}$ vs. 4 ). In trial NB90, additional clinical risk factors (serum LDH elevation, age $\geq 9$ months, and no more than biopsy of the primary tumor) discriminated stage 3 into $\mathrm{A} / \mathrm{B}$ or $\mathrm{C} / \mathrm{D}$ (Table $\mathrm{S} 1$ in the Electronic Supplementary Material [ESM]). From trial NB90 onwards, MYCN amplification qualified a patient for the high-risk group regardless of the stage. In trial NB2004, chromosome 1p aberrations (deletion, imbalance) of stage 2 and 3 tumors resulted in an upstaging from the observation to the intermediate-risk group. Infants with stage 4 neuroblastoma were treated as high risk in the NB79-NB97 trials and as intermediate risk in the NB2004 trial.

For the survival analysis, the high-risk group was confined to stage 4 and aged $\geq 18$ months at diagnosis or to $M Y C N$-amplified neuroblastoma at any stage and any age.

\subsection{Staging}

Evans' staging system [10] was used for trials NB79-NB85 (Roman numerals) and the International Neuroblastoma Staging System (INSS) [6] for trials NB90-NB2004 (Arabic numerals). For simplicity, both staging systems are reported in Arabic numerals in this article. Throughout all the trials for stage $4 \mathrm{~S}$, the German neuroblastoma group accepted larger primary tumors (stage 3 tumors) and unusual metastatic sites (e.g., distant lymph nodes, intracerebral lesions, single small mIBG osteomedullary uptake spots in otherwise typical stage $4 \mathrm{~S}$ disease pattern). This differs from the strict $4 \mathrm{~S}$ definitions but is regarded as compatible with its basic biological character of regression and remained constant over the study period (except for the extension of the age from 12 to 18 months in trial NB2004). Compared with other series, the German stage $4 \mathrm{~S}$ category included a portion of infants with stage 4 characteristics as defined by INSS. Infants with unambiguous bony metastases were always classified as stage 4 .

\subsection{Remission Criteria}

The INSS remission status [6] was used prospectively for trials NB90-NB2004. For patients in trials NB79-NB85, only the categories "complete remission" (CR; complete tumor disappearance), "partial remission" (PR; $>50 \%$ disappearance, but detectable residuals), "no remission" ( $<50 \%$ disappearance), and "progression" (enlarged or new tumor) were available. The remission status was evaluated after three cycles in trials NB79-85, after four cycles in trials NB90-97, and after two cycles in trial NB2004.

\subsection{Neuroblastoma Screening}

Urine screening for catecholamine metabolites to detect early neuroblastoma was offered to children at approximately 1 year of age in 6 of 16 German States from 1995 to 2000 [11].

\subsection{Structure of Chemotherapy Cycles and Measurement of Time Intervals}

Details of the designations and the structure of chemotherapy cycles are given in Table S1 in the ESM.

Time intervals between chemotherapeutic cycles were considered as indirect measurements of dose intensity and toxicity. This is possible because the criteria to start the next cycle were roughly stable from trial to trial.

\subsection{Surgical Therapy}

Complete tumor resection or maximal debulking were intended, but risky procedures or mutilations (e.g., nephrectomy) had to be avoided. Complete resection was defined as macroscopical complete tumor removal, allowing microscopic residuals. Incomplete resection comprised all conditions with macroscopic residual tumor described by the surgeon or the radiologist. Biopsy refers to sampling of tumor tissue without removal of a substantial portion of the tumor. Although the aggressiveness of the surgical approach decreased over the decades, the removal of a vital tumor (actively accumulating contrast medium or mIBG) was recommended throughout all trials.

\subsection{Radiotherapy}

Radiotherapy was applied to high-risk cases with unresected primary tumors that presented as vital at the end of induction chemotherapy or during consolidation treatment (Table S4 in the ESM). In trial NB90, up to four bony metastases were additionally irradiated. In trials NB97 and NB2004, mIBG radiotherapy and percutaneous irradiation were used as part of the myeloablative regimen if patients had mIBG-positive lesions (primary tumors and/or metastases) at the end of induction chemotherapy. In trial NB85, patients received three cycles of frontline mIBG therapy without stem cell rescue. 


\subsection{Treatment of Low- and Intermediate-Risk Patients}

Therapeutic principles for patients with low- and intermediate-risk disease are outlined in Tables S2 (stages 1-3) and S3 (stage 4S) in the ESM. In general, therapeutic restraint was increasingly applied in view of the growing recognition of a significant potential for spontaneous regression. Initial surgical intervention was always recommended to remove a localized tumor or at least to obtain biopsy tissue for histological and molecular diagnosis. Front-line chemotherapy was recommended for stage 1 tumors only if they were $M Y C N$ amplified.

Stage 2 neuroblastomas with regional lymph node involvement received reduced chemotherapy in trials NB85 and NB90. In trial NB97, unresected stage 2 tumors received four cycles of chemotherapy. In trial NB2004, chemotherapy was given only to patients with organ- or life-threatening symptoms (until relief).

Patients with stage 3 tumors were considered to have "advanced disease" in the 1980s and early 1990s and were treated aggressively. This was in contrast to an observation strategy of patients aged $0-1$ year with stage 3 in trials NB97 and NB2004. Patients with stage 3 (patients aged $\geq 2$ years, $1 \mathrm{p}$ aberration, and/or $M Y C N$ amplification) were treated in the intermediate- or high-risk group.

Infants with stage $4 \mathrm{~S}$ neuroblastoma (Table S3 in the ESM) who presented with organ- or life -threatening symptoms received immediate chemotherapy to alleviate the symptoms and to induce regression. Chemotherapy was stopped as soon as symptoms improved or regression was evident.

\subsection{Treatment of High-Risk Patients}

The different treatment programs for patients with highrisk neuroblastoma are shown in Fig. S1 in the ESM. Details of the chemotherapy cycles and of other treatment elements are given in Tables S2 and S4, respectively, in the ESM. After diagnosis, cyclic chemotherapy was given to relieve symptoms and to induce remission. If a residual tumor was present after induction chemotherapy, surgical resection, or, in case of unresectability, radiotherapy was recommended. Subsequent consolidation therapy varied over time and included interferon $\beta$, anti-GD2 antibody, myeloablative therapy with autologous stem cell transplantation, maintenance chemotherapy, and retinoic acid.

\subsection{Late Sequelae}

Late sequelae were defined as side effects reported to the trial office more than 1 year after diagnosis and excluded short-term toxicities. Second malignancies were included at any time without restriction from diagnosis to the end of follow-up or to the data lock, whichever occurred first. The grading system of common terminology criteria for toxicity was not applied throughout all the trials because it limited the analysis. Local physicians reported what they regarded as "significant" for the patients during trials NB79-NB85. Trials NB90 and NB97 used the common World Health Organization (WHO) toxicity criteria [12], and trial NB2004 used the Common Terminology Criteria for Adverse Events (CTCAE) version 3.0 criteria [13]. Grades 2-4 were regarded as significant. Only ototoxicity was evaluated according to the Brock criteria [14], and Brock grades 3-4 were regarded as significant.

\subsection{Statistical Analysis}

Age standardized incidence rates (ASRs) were computed using the Segi-world standard. Trend analyses were performed using Poisson log-linear modeling with age group indicators and an indicator for the screening period in the model. An estimated rate ratio (RR) of the deviation of the screening period from the overall trend is given, as well as an RR for trend per 10 calendar years. For "year", the default model was untransformed, and transformations were done as described in Royston and Sauerbrei [15]. When a transformation was a better fit, RRs were given for the comparison of 2010 versus 2000 (screening adjusted). RRs are supplemented with a 95\% confidence interval (CI).

The IBM SPSS statistic package version 24 was used for the analyses. Frequencies were analyzed using the $\chi^{2}$ test according to Pearson. For time trends of frequency estimations, the Jonckheere-Terpstra and the Cochrane-Armitage tests were employed. For comparison of the Kaplan-Meier survival estimations, the log rank test was applied. Event-free survival (EFS) was calculated from the day of histological diagnosis to an event or date of last information if no event occurred. Event was defined as tumor progression or death for any reason for EFS and death for any reason for overall survival (OS).

\section{Results}

\subsection{Time Trends of Incidence and Relative Frequency of Participants in Clinical Trials}

The population-based median annual age standardized incidence rate (ASR) of neuroblastoma per million children per year in Germany between the periods 1980 and 2015 was 13.2 (minimum 8.7 [1981], incomplete registration; maximum 17.8 [2000], last year of screening trial). For stages 1-3, the median ASR was 6.3 (minimum 3.3 [1981]; 


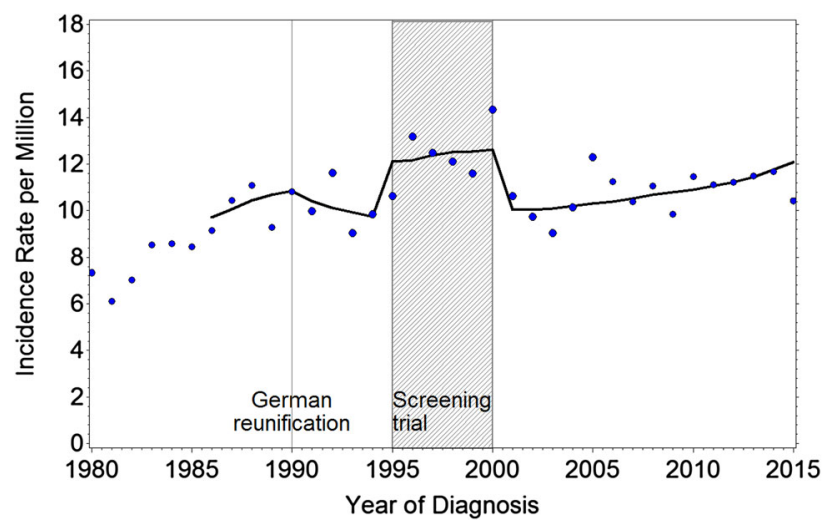

Fig. 1 Incidence rates of neuroblastoma per million children in Germany 1980-2014 aged 0-14 years. Trend fitted 1986-2014, adjusted for population age distribution, German reunification, and screening trial

maximum 9.5 [2000]), and for stage 4, it was 4.6 (minimum 2.6 [1985]; maximum 6.6 [2000]).

The age-adjusted regression curve (Fig. 1) shows an increase in crude neuroblastoma incidence rates during the period of neuroblastoma screening (RR 1.26; 95\% CI $1.17-1.36$ ), with a $7 \%$ increase per 10 years for neuroblastoma (RR/10 years 1.07 ; $95 \%$ CI $1.03-1.12$; data adjusted for the screening period). Much of the increase resulted mainly from stage 1-3 diagnoses (RR during screening $1.40 ; 2010$ vs. 2000 RR 1.10; 95\% CI 1.04-1.15). This was particularly evident for the group aged 1 to $<2$ years for stage $1-3$ tumors, which showed a screening-adjusted increase of $20 \%$ from 2000 to 2010 (RR 2010 vs. 2000 1.20; 95\% CI 1.03-1.40). The incidence of neuroblastoma stage $4 \mathrm{~S}$ ( $<1$ year of age) increased by $14 \%$ (RR 2010 vs. 2000 1.14; 95\% CI 1.07-1.21), with a flattening curve in recent years. No significant change was observed over time for the total group of children with stage 4 neuroblastoma, neither during the screening period nor afterwards (RR 2010 vs. $2000 \quad 0.98,95 \%$ CI 0.91-1.06). Among stage 4 patients, patients aged $<1$ year showed a small increase of $3 \%$ over a 10 -year period (RR $1.03 ; 95 \%$ CI $0.93-1.14)$ and for the children aged 1 to $<2$ years, a $12 \%$ decrease in neuroblastoma incidence per 10-year period (RR 0.88; 95\% CI 0.75-1.03) was observed. Overall, no change in incidence of stage 4 patients was seen, whereas an increase in localized stages and stage $4 \mathrm{~S}$ was observed.

The percentage of patients who were resident in Germany, known to the nationwide German Childhood Cancer Registry during the periods 1980-2015 and participated in the neuroblastoma trials was $96.6 \%$. The coverage was less during the early 1980 s, reached more than $90 \%$ during the period 1985-1994, and was consistently $\geq 98 \%$ from 1995 to 2015 .
Table 1 Stage distribution by trial

\begin{tabular}{lrlll}
\hline Trial & \multicolumn{1}{l}{$N^{\mathrm{a}}$} & Stages $1-3(\%)$ & Stage 4 S $(\%)$ & Stage 4 (\%) \\
\hline NB79 & 188 & 42.5 & 10.6 & 46.8 \\
NB82 & 215 & 47.4 & 09.8 & 42.8 \\
NB85 & 405 & 48.9 & 09.1 & 42.0 \\
NB90 & 933 & 48.2 & 10.1 & 41.7 \\
NB97 & 1200 & 57.9 & 09.9 & 32.2 \\
NB2004 & 1518 & 50.4 & 12.6 & 37.0 \\
NB79-2004 & 4459 & 51.3 & 10.8 & 37.8 \\
\hline
\end{tabular}

a Stage information not available for four patients

b Neuroblastoma screening (1995-2000) and infants' regression observation trial (1995-2004) had an influence on the stage distribution of the NB97 trial

\subsection{Frequencies of Stages, Age at Diagnosis, and Time to Diagnosis}

A total of 4463 patients were enrolled into one of the nationwide trials between 1979 and 2015. Table 1 shows the number of patients in the trials and the relative stage distribution. Half of the cohort $(51 \%)$ had localized/regional neuroblastoma stages (1-3), roughly $11 \%$ stage 4 S, and $38 \%$ stage 4 disease. The proportion of stage 1-3 increased during the neuroblastoma screening period [11].

Patients with neuroblastoma were diagnosed at an earlier age in more recent years (Table 2). The median age at diagnosis decreased from 23 months during 1980-1984 to 15 months during 2010-2014. The drop occurred continuously but was particularly evident during and following the neuroblastoma screening period (NB97 and NB2004). The median age at diagnosis is now 10 months for stages 1-3 and 32 months for stage 4 (stage 4 restricted to $\geq 18$ months: 39 months). The median age at diagnosis of stage $4 \mathrm{~S}$ disease was 2.5 months for all 482 registered patients.

The time from the first reported symptom to the final neuroblastoma diagnosis was 13 days in stage $4 \mathrm{~S} ; 21$ days in low- and intermediate-risk disease; 30 days in stage 4 cases aged $\geq 18$ months; and 24 days for all patients. Asymptomatic cases were excluded for this calculation. The frequency of asymptomatic cases, found during routine examinations of the health status or for unrelated medical conditions, increased continuously from 12\% (1980-1985) to a maximum of 44\% during 1995-1999 (main screening period). From 2000 to 2014, the frequency of patients without symptoms remained high (34-36\%).

\subsection{Frequencies of Primary and Metastatic Sites}

The percentage of patients with an unknown primary tumor site decreased from 4.2 to $0.8 \%$ (Table S6 in the ESM). A total of $50 \%$ of the primaries were found at the adrenal site, 
Table 2 Median age (months) at diagnosis by time period, 1980-2014

\begin{tabular}{|c|c|c|c|c|c|c|c|c|c|}
\hline \multirow[t]{2}{*}{ Period } & \multirow[t]{2}{*}{$N$} & \multicolumn{2}{|c|}{ Stages $1-3$ and 4 aged $<18$ mo } & \multicolumn{2}{|c|}{ Stage $4 \mathrm{~S}$} & \multicolumn{2}{|c|}{ Stage 4 aged $>18 \mathrm{mo}$} & \multicolumn{2}{|l|}{ All } \\
\hline & & Median & Min-max & Median & Min-max & Median & Min-max & Median & Min-max \\
\hline 1980-84 & 334 & 13.0 & $0-207$ & 1.6 & $0.1-8$ & 40.0 & $19.4-242$ & 22.7 & $0-242$ \\
\hline 1985-89 & 413 & 13.3 & $0-231$ & 2.0 & $0-15$ & 38.6 & $18.2-143$ & 19.0 & $0-231$ \\
\hline 1990-94 & 653 & 11.8 & $0-232$ & 1.9 & $0-11$ & 39.6 & $18.2-215$ & 17.8 & $0-232$ \\
\hline 1995-99 & 820 & 13.5 & $0-206$ & 2.1 & $0-11$ & 43.5 & $18.3-250$ & 15.1 & $0-250$ \\
\hline $2000-04$ & 715 & 11.4 & $0-253$ & 2.9 & $0-10$ & 40.8 & $18.8-247$ & 15.0 & $0-253$ \\
\hline 2005-09 & 684 & 11.4 & $0-248$ & 3.9 & $0-16$ & 42.1 & $18.3-255$ & 16.1 & $0-255$ \\
\hline 2010-14 & 665 & 10.3 & $0-306$ & 2.7 & $0-12$ & 39.2 & $18.4-251$ & 15.1 & $0-306$ \\
\hline All & 4284 & 12.2 & $0-306$ & 2.5 & $0-16$ & 40.6 & $18.2-255$ & 16.1 & $0-306$ \\
\hline Jonckheere-Terpstra test & & \multicolumn{2}{|l|}{$P<0.001$} & \multicolumn{2}{|c|}{$P=0.005$} & \multicolumn{2}{|c|}{$P=0.947$} & \multicolumn{2}{|c|}{$P<0.001$} \\
\hline
\end{tabular}

Table 3 Frequencies of $M Y C N$ amplification in 3251 patients with neuroblastoma ${ }^{a}$ by trial

\begin{tabular}{|c|c|c|c|c|c|c|c|c|c|c|c|c|c|}
\hline \multirow[t]{2}{*}{ Trial } & \multirow[t]{2}{*}{$N$} & \multicolumn{2}{|l|}{ Stage $1-3$} & \multicolumn{2}{|c|}{ Stage $4 \mathrm{~S}$} & \multicolumn{2}{|c|}{$\begin{array}{l}\text { Stage } 4 \text { and aged } \\
<18 \text { mo }\end{array}$} & \multicolumn{2}{|c|}{$\begin{array}{l}\text { Stages } 1-3 / 4 \mathrm{~S} / 4 \text { aged } \\
<18 \mathrm{mo}\end{array}$} & \multicolumn{2}{|c|}{$\begin{array}{l}\text { Stage } 4 \text { and aged } \\
\geq 18 \text { mo }\end{array}$} & \multicolumn{2}{|l|}{ All } \\
\hline & & $n / N$ & $\%$ & $n / N$ & $\%$ & $n / N$ & $\%$ & $n / N$ & $\%$ & $n / N$ & $\%$ & $n / N$ & $\%$ \\
\hline NB90 & 630 & $30 / 301$ & 10.0 & $8 / 62$ & 12.9 & $25 / 65$ & 38.5 & $63 / 428$ & 14.7 & $60 / 202$ & 29.7 & $123 / 630$ & 19.5 \\
\hline NB97 & 1174 & $56 / 684$ & 8.2 & $10 / 111$ & 9.0 & $41 / 101$ & 40.6 & $107 / 896$ & 11.9 & $75 / 278$ & 27.0 & $182 / 1174$ & 15.5 \\
\hline NB2004 & 1447 & $59 / 714$ & 8.2 & $21 / 175$ & 12.0 & $52 / 130$ & 40.0 & $132 / 1019$ & 13.0 & $150 / 428$ & 35.0 & $282 / 1447$ & 19.5 \\
\hline All & 3251 & $145 / 1699$ & 8.5 & $39 / 348$ & 11.2 & $118 / 296$ & 39.9 & $302 / 2343$ & 12.9 & $285 / 909$ & 31.3 & $587 / 3251$ & 18.1 \\
\hline
\end{tabular}

$n$ number of patients with $M Y C N$ amplification per group, $N$ number of patients per group (stage, age)

${ }^{\text {a }}$ MYCN data missing in additional 398 patients (10.9\%)

$29 \%$ at other abdominal sites (including pelvic), $16 \%$ at thoracic sites, and $4 \%$ at cervical sites. The order of the frequencies for the primary sites remained stable throughout the trials.

The frequencies of metastatic sites are listed in Table S7 in the ESM. Stage 4 patients aged $\geq 18$ months presented with bone marrow metastases in $89 \%$ and bone metastases in $65 \%$ of cases. Lymph nodes were involved in $21 \%$ of cases, liver in $13 \%$, intracranial sites in $6 \%$, lungs or pleura in $5 \%$, and skin in $<1 \%$. In total, $95 \%$ of all patients had metastases in the bone marrow and/or bones (osteomedullary). This figure was remarkably stable over the trials (range 92-98\%). Time trends were observed for an increased detection of metastases in bone marrow (by cytology plus imaging techniques) and in lung/pleura sites. A decreasing frequency was seen for liver metastases and bone lesions.

\subsection{Frequencies of Risk Factors}

Determination of $M Y C N$ amplification has been recommended as the initial diagnostic tool for neuroblastoma since 1990. In total, 9\% of patients with localized neuroblastoma, $10 \%$ with stage $4 \mathrm{~S}$ disease, and $40 \%$ of children with stage 4 aged $<18$ months had $M Y C N$-amplified neuroblastoma (Table 3). The combined low- and intermediate-risk group (stages 1-3 all ages, stage $4 \mathrm{~S}$, stage 4 aged $<18$ months) had MYCN amplification in $13 \%$ of the cases. Clinical high-risk neuroblastoma (stage 4 aged $\geq 18$ months) showed MYCN amplification in $31 \%$ of cases. In this subgroup, an increase from trial NB97 (27\%) to trial NB2004 (35\%) was observed $(p=0.026)$. All other differences were not statistically significant.

Table 4 demonstrates that abnormal elevations of LDH (in $60 \%$ of the patients), ferritin (in 36\% of the patients), and chromosome $1 \mathrm{p}$ aberrations (in $28 \%$ of the patients) were found across all patients. Children with low- and intermediate-risk disease (stages 1-3, stage $4 \mathrm{~S}$, stage 4 aged $<18$ months) had fewer LDH abnormalities (46\%), ferritin elevations (24\%), and chromosome $1 \mathrm{p}$ aberrations (21\%) than patients with stage 4 aged $\geq 18$ months (LDH abnormal in $92 \%$, ferritin in $64 \%$, and chromosome $1 \mathrm{p}$ aberrant in 50\%). With the exception of the screening period that included more low-risk patients (trial NB97), the proportions of abnormal LDH, ferritin, and chromosome $1 \mathrm{p}$ aberrations did not differ between the studies, indicating that the risk profile of the patients with neuroblastoma was mostly constant throughout the trials. 
Table 4 Frequency of risk factors in neuroblastoma cases (all stages) by trial

\begin{tabular}{|c|c|c|c|c|c|c|}
\hline \multirow[t]{2}{*}{ Trial } & \multicolumn{2}{|c|}{ LDH abnormal } & \multicolumn{2}{|c|}{ Ferritin abnormal } & \multicolumn{2}{|c|}{$1 \mathrm{p}$ aberration or $1 \mathrm{p}$ imbalance } \\
\hline & $n / N$ & $\%$ & $n / N$ & $\%$ & $n / N$ & $\%$ \\
\hline NB79 & $62 / 84$ & 73.8 & $2 / 5$ & 40.0 & NA & \\
\hline NB82 & $122 / 181$ & 67.4 & $59 / 151$ & 39.1 & NA & \\
\hline NB85 & $222 / 349$ & 63.6 & $128 / 312$ & 41.0 & NA & \\
\hline NB90 & $557 / 875$ & 63.7 & $270 / 749$ & 36.0 & $100 / 325$ & 30.8 \\
\hline NB97 & $611 / 1132$ & 54.0 & $292 / 926$ & 31.5 & $212 / 842$ & 25.2 \\
\hline NB2004 & $858 / 1432$ & 59.9 & $414 / 1110$ & 37.3 & $399 / 1344$ & 29.7 \\
\hline All & $2432 / 4053$ & 60.0 & $1165 / 3253$ & 35.8 & $711 / 2511$ & 28.3 \\
\hline Cochrane-Armitage test & $<0.001$ & & 0.292 & & 0.512 & \\
\hline
\end{tabular}

$L D H$ lactate dehydrogenase, $n$ number of patients with abnormal values compared to age-related reference values, $N$ number of patients with available data, $N A$ not applicable

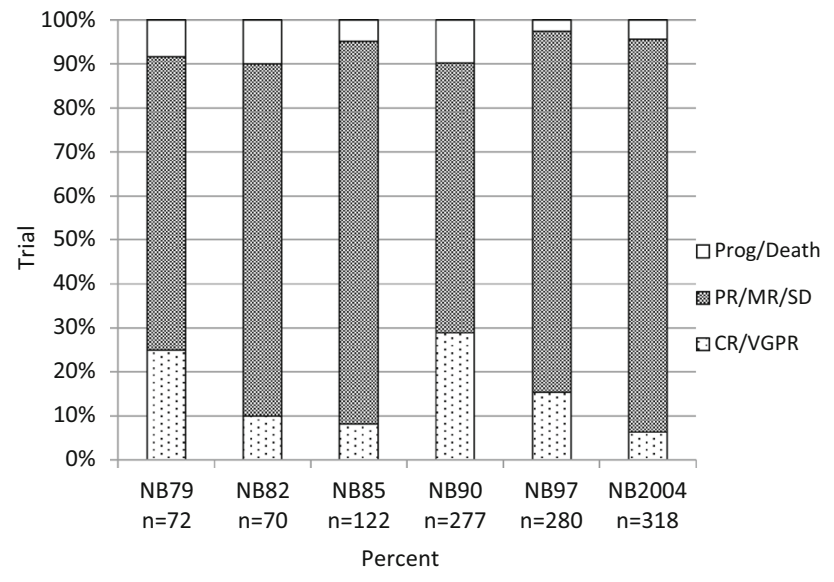

Fig. 2 Frequencies of achieved remission categories after induction chemotherapy in 1139 patients with stage 4 neuroblastoma aged $\geq 18$ months by trial. $C R$ complete remission, $M R$ mixed response, $P R$ partial remission, $S D$ stable disease, $V G P R$ very good partial remission

\subsection{Remission Proportions after Induction Chemotherapy}

Figure 2 shows the categories of achieved remission after induction chemotherapy for patients with stage 4 disease who were aged $\geq 18$ months by trial. Considering all trials together, $16 \%$ of patients achieved complete or very good partial remission after initial chemotherapy (range 6-29\%), and $78 \%$ achieved partial or mixed remission or stable disease (range 61-89\%). In 6\% (range 2-10\%), the tumor progressed or death occurred. Considerable fluctuations between the trials were observed; however, trends for improvements over time were not detected.

\subsection{Frequencies of Extent of Primary Tumor Resections}

Complete macroscopic removal with and without microscopic residuals was achieved in $64 \%$ of 3985 evaluable patients (all stages, best result counted in case of more than one operation). A total of $22 \%$ had an incomplete resection at best, and 14\% of patients had a biopsy. Comparing trials NB97 and NB2004, the number of complete resections decreased (from 70 to 55\%), whereas the proportion of incomplete resections increased (from 20 to $23 \%$ ) and the proportion of biopsies increased (from 10 to $22 \%$ ).

Details of the resectability for the regional stages 1, 2, 3, and for stage $4 \mathrm{~S}$ are not presented in this report because of the impact of spontaneous regression on the surgical decision. For patients with stage 4 disease aged $\geq 18$ months, in $63 \%$ of the patients, the primary tumor was completely removed; in $27 \%$, there was incomplete resection; and in $10 \%$ of the patients, only a biopsy was performed. Complete resections reached a maximum of $75 \%$ in trial NB97 (incomplete resections 18\%, biopsies $7 \%$ ) but decreased in the most recent trial NB2004 (58\% complete, 32\% incomplete, 9\% biopsy) (Fig. 3).

\subsection{Frequencies of Sparing Chemotherapy for Patients with Low- and Intermediate-Risk Disease}

The concept of avoiding chemotherapy has been applied to an increasingly greater number of patients (Tables S2 and $\mathrm{S} 3$ in the ESM). Figure 4 shows the increasing percentage of patients who never received chemotherapy, neither at initial treatment nor, in the event of recurrence or progression, for second-line therapy. In trials NB79 and NB82, $17 \%$ of patients with stage $4 \mathrm{~S}$ and $35 \%$ of stage $1-3$ disease (all ages) received no chemotherapy. The figures increased to $41 \%$ for stage $4 \mathrm{~S}$ and to $60 \%$ for stages $1-3$ (NB2004).

\subsection{Event-Free and Overall Survival}

The 10-year EFS of all 4463 patients was 57\%, increasing from $44 \%$ (NB79) to $55 \%$ (NB2004). The 10-year OS was 


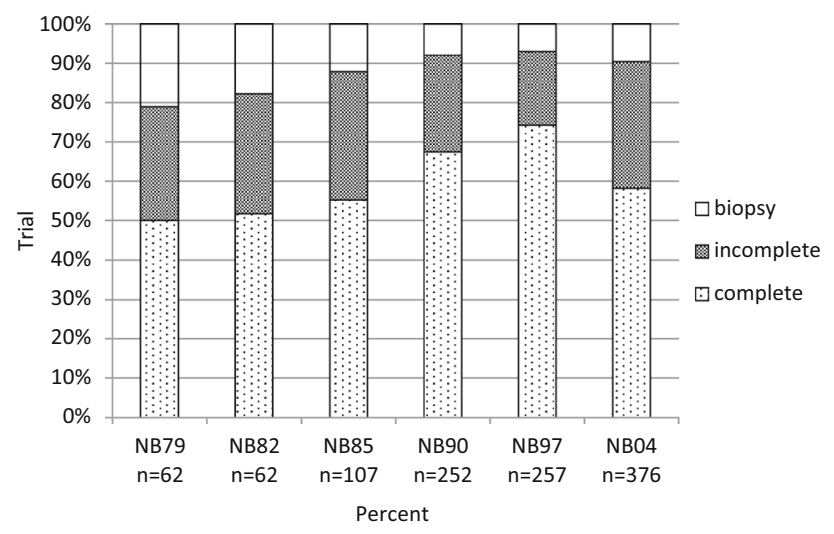

Fig. 3 Distribution of grades of surgical resection by trial in 1116 patients with stage 4 neuroblastoma aged $\geq 18$ months by trial. complete macroscopic complete resection with and without microscopic residuals, incomplete macroscopic incomplete resection, biopsy only biopsy material obtained, no tumor resection. If more than one surgical intervention was performed, the best achieved result counted

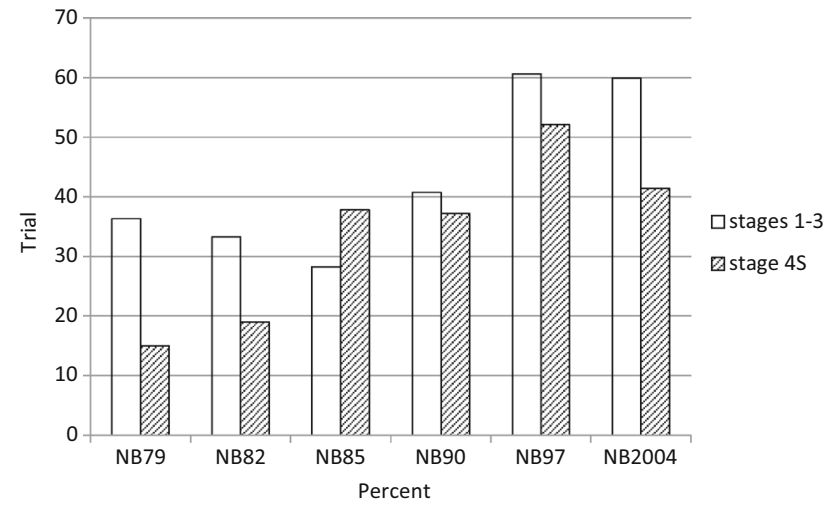

Fig. 4 Patients without chemotherapy (not after initial diagnosis or after recurrence) (including intermediate-risk patients and children with recurrence therapy). Stages 1-3 (low and intermediate risk, $n$ / $N=1130 / 2290)$ and $4 \mathrm{~S}(n / N=193 / 482)$

$67 \%$ for all patients, increasing from $46 \%$ (NB79) to $72 \%$ (NB2004) (Fig. 5a, b; Tables S8 and S9 in the ESM).

\subsubsection{Survival by Stage}

Children with localized neuroblastoma (stages 1-3) had similar rates of EFS throughout the trials. OS increased by roughly $8 \%$ (Table S9 in the ESM), although the therapeutic intensity decreased (Table S2 in the ESM).

Infants with stage $4 \mathrm{~S}$ had a 10 -year EFS of $74 \%$ and a 10 -year OS of $85 \%$. The lowest 10-year EFS was $67 \%$ (NB82), and the highest was 76\% (NB97 and NB2004). The 10-year OS proportions increased from $71 \%$ (NB82) to 87\% (NB2004) despite reduced chemotherapeutic intensity (Table S3 in the ESM).

Stage 4 patients aged $\geq 18$ months with and without MYCN amplification improved from a 3\% 10-year EFS
(NB79) to 29\% (NB97) and 24\% (NB2004). The 10-year OS increased from 2\% (NB79) to $33 \%$ (NB97) and $38 \%$ (NB2004) (Fig. 5c, d; Tables S8 and S9 in the ESM).

\subsubsection{Survival by MYCN Amplification}

Figure 5e, f demonstrate that the outcome was inferior for patients with $M Y C N$ amplification and stage 4 aged $\geq 18$ months (10-year EFS 21\%, 10-year OS 26\%) compared with stages $1,2,3,4 \mathrm{~S}$, and 4 aged $<18$ months (10year EFS 37\%, 10-year OS 48\%) (log-rank test for EFS $p=0.005$, for $\mathrm{OS} p=0.002)$. In the $M Y C N$-amplified group, the EFS was not influenced by single stages 1, 2, 3, and $4 \mathrm{~S}$ (range 40-51\%), with the exception of patients with stage 4 disease aged $<18$ months, who had a lower 10 -year EFS (29\%) (Table S8 in the ESM). In contrast, OS was stage dependent in $M Y C N$-amplified cases: the 10-year OS was $92 \%$ for stage $1,57 \%$ and $56 \%$ for stages 2 and 3, $41 \%$ for stage $4 \mathrm{~S}$, and $37 \%$ for stage 4 aged $<18$ months (logrank $p<0.001$ ) (Table S9 in the ESM). Notably, the frequency of MYCN amplification in the group of patients with stage 4 aged $<18$ months was $40 \%$ (Table 3 ).

If the $M Y C N$ oncogene was non-amplified, children with stage 4 aged $>18$ months had clearly inferior EFS and OS compared with all other stage/age groups (Fig. S2, Tables S8 and S9 in the ESM). Recurrences or progression were not necessarily fatal in these cohorts, e.g., stage 3 neuroblastoma without MYCN amplification had a 10-year EFS of $64 \%$ and a 10 -year OS of $86 \%$.

\subsubsection{Survival by the Grade of Remission in Stage 4 Aged $\geq 18$ Months and High-Risk Patients}

The impact of remission categories on survival is shown in Fig. $6 \mathrm{a}, \mathrm{b}$ for the 1139 patients with stage 4 neuroblastoma aged $\geq 18$ months (all trials NB79-NB2004). Children with partial remission/mixed response/stable disease (PR/MR/ SD) had a 10-year EFS of $21 \%$ compared with $32 \%$ for those with complete remission (CR)/very good partial remission (VGPR) (log-rank test $p=0.002$ ). The 10-year OS figures were $27 \%$ for $\mathrm{PR} / \mathrm{MR} / \mathrm{SD}$ and $36 \%$ for CR/ VGPR (log-rank test $p=0.021$ ).

The influence of the remission status on the survival was trial dependent and detectable for trials NB90 and NB2004 on EFS and for trials NB90, NB97, and NB2004 on OS. No impact on EFS was seen for trials NB79, NB82, NB85, and NB97, and no impact on OS for trials NB79, NB82, and NB85.

Data for detailed remission categories were available from the year 1990 onwards. The CR group was best for EFS and OS but was small (6.1\% of patients). The EFS and OS curves did not differ from the CR and VGPR groups in trials NB90 and NB 2004. Only in trial NB97 did patients with CR have a better EFS and OS than patients with VGPR. Categories MR 
Fig. 5 Kaplan-Meier estimates of $\mathbf{a}, \mathbf{b}$ all patients with neuroblastoma (NB79-NB2004; $n=4463$ ) by trial: a event-free survival, b overall survival; c, d stage 4 aged $\geq 18$ months at diagnosis (NB79-NB2004; $n=1276$ ) by trial: c event-free survival, d overall survival; e, f $M Y C N$-amplified neuroblastoma by stage and age (NB90-NB2004; $n=586$ ): e event-free survival, f overall survival. Log-rank tests, af $p<0.001$

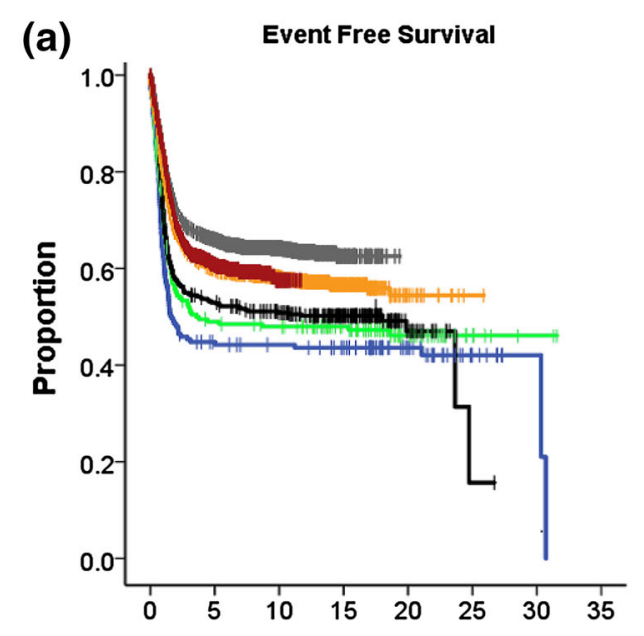

(c)

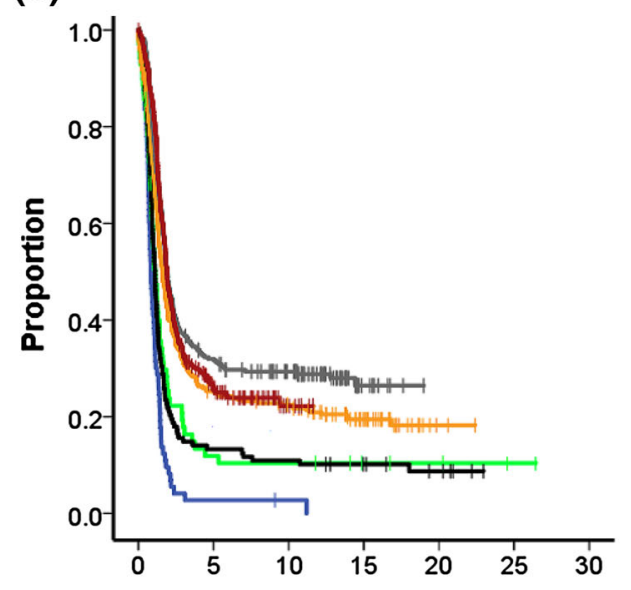

(e)

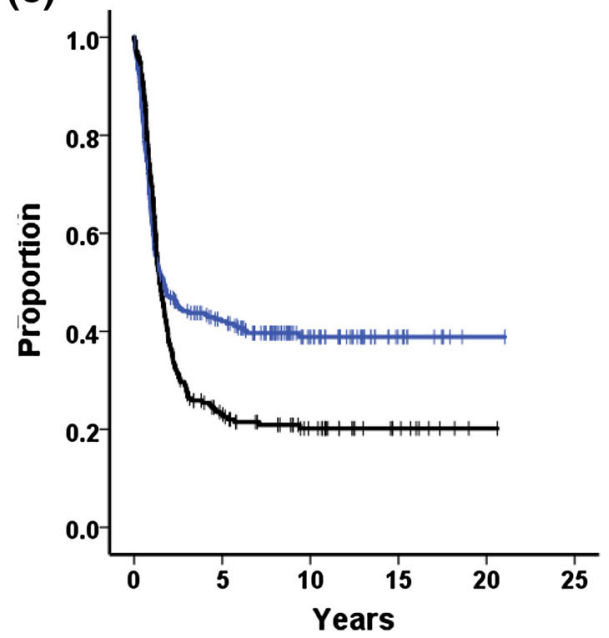

(b)

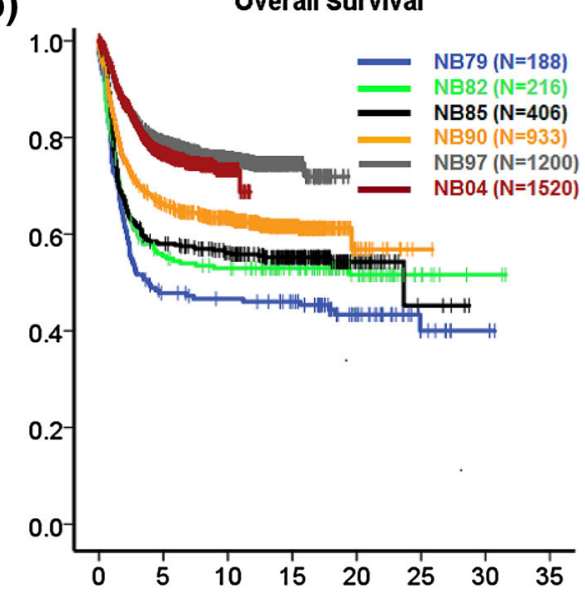

(d)

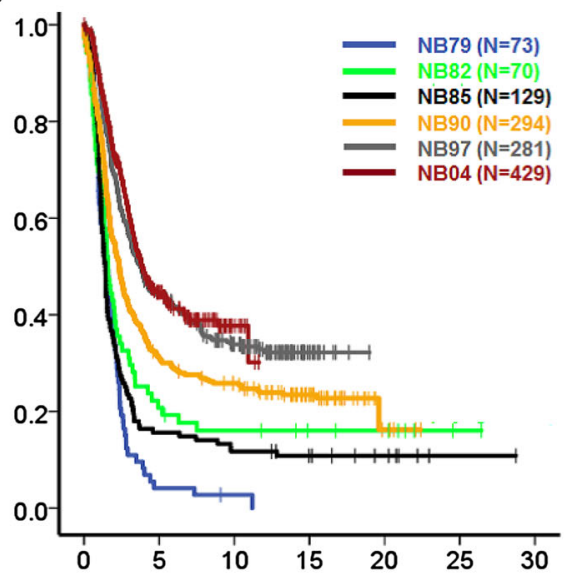

(f)

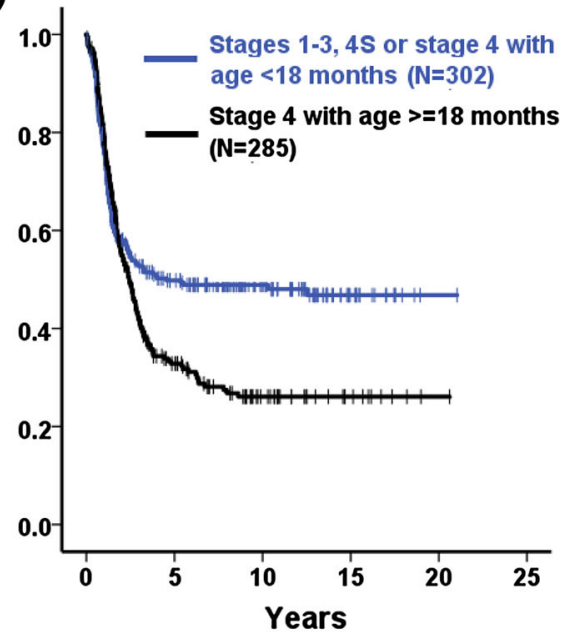

(2.4\% of all patients) and SD (3.3\% of all patients) were grouped together with the PR category (68.7\%).

The high-risk group yielded a similar result: patients with $\mathrm{PR} / \mathrm{MR} / \mathrm{SD}$ had an inferior EFS and OS compared with patients with CR/VGPR $(n=1126$; NB90, NB97, NB2004, log-rank $p<0.0001)$.
3.8.4 Survival by the Grade of Primary Tumor Resection in Stage 4 Patients Aged $\geq 18$ Months

Figure $6 c, d$ demonstrate that if "biopsy" was the best achieved operation result in patients with stage 4 disease aged $\geq 18$ months, the 10 -year EFS (10\%) and 10-year OS 
Fig. 6 Kaplan-Meier estimates of patients with neuroblastoma stage 4 aged $\geq 18$ months (NB79-NB2004; $n=1139$ ) by remission categories $(\mathrm{a}, \mathrm{c})$ and by grade of surgical resection $(b, d)$ : a event-free survival (logrank test complete remission/ very good partial remission vs. partial remission/mixed response/stable disease $p=0.002)$, c overall survival (log-rank test complete remission/very good partial remission vs. partial remission/ mixed response/stable disease $p=0.021)$; stage 4 aged $\geq 18$ months by best achieved grade of surgical resection (NB79-NB2004; $n=1116)$ : $\mathbf{b}$ event-free survival (log-rank test biopsy vs. incomplete $p<0.001$; incomplete vs. complete $p=0.807)$, $\mathbf{d}$ overall survival (log-rank test biopsy vs. incomplete $p<0.001$; incomplete vs. complete $p=0.270)$. $C R$ complete remission, $M R$ mixed response, $P R$ partial remission, $S D$ stable disease, $V G P R$ very good partial remission

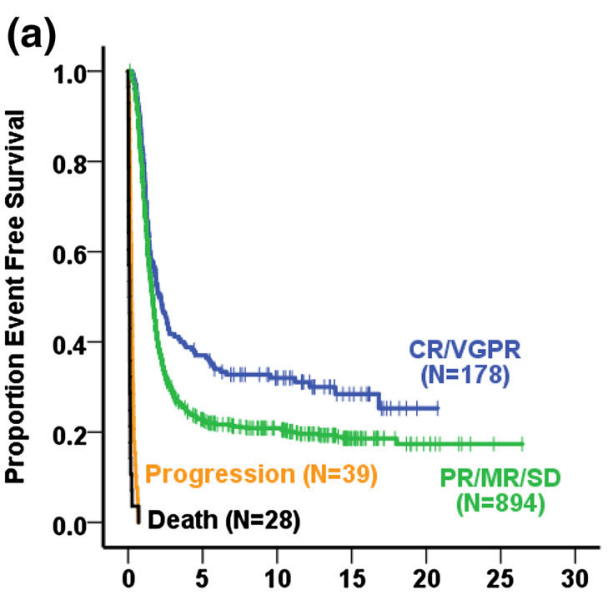

(b)

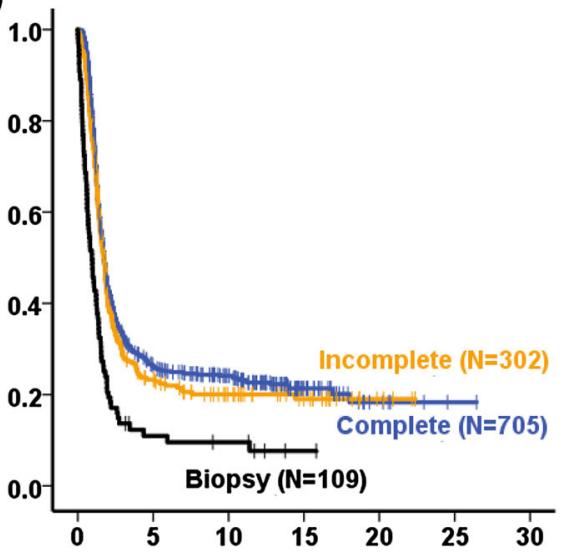

(c)

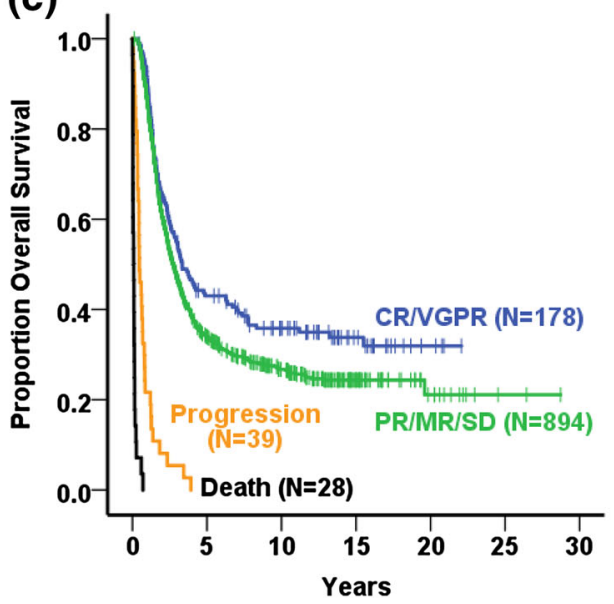

(d)

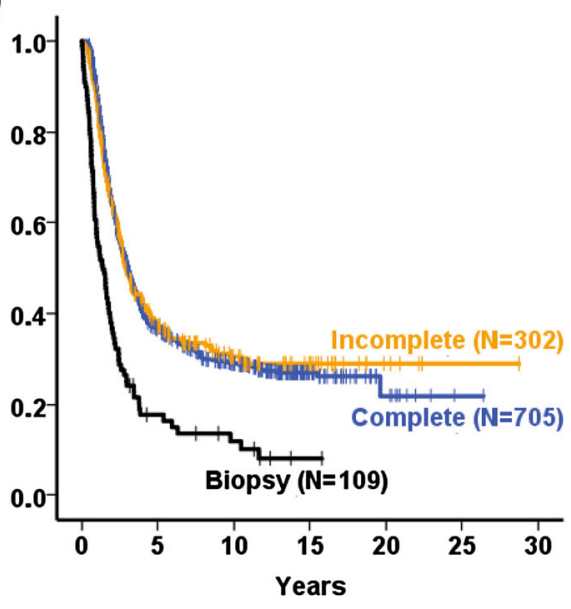

$(12 \%)$ were inferior compared with those with higher degrees of resection (incomplete removal: 20\% EFS, $30 \%$ OS; macroscopically complete removal 24\% EFS, $29 \%$ OS). Complete versus incomplete removal was not statistically different in the total stage 4 aged $\geq 18$ months group or in single trials, with one exception: In the NB82 trial, complete resection $(n=32)$ had a better EFS and OS than did incomplete resection $(n=19)$ (log-rank for EFS $p=0.017$, for OS $p=0.025)$.

\subsection{Toxicity}

\subsubsection{Frequencies of Death from Toxicity}

Table 5 shows a proportion of $6 \%$ for toxic death during the time of protocol therapy for patients with stage 4 neuroblastoma aged $\geq 18$ months (maximum was $11.6 \%$ in trial NB85, minimum was $3.8 \%$ in trial NB2004). Most toxic deaths in this group were caused by chemotherapy (including myeloablative chemotherapy; 88\%), some by surgical complications (9\%), and rarely by other causes $(3 \%)$. Children with stage $1,2,3,4 \mathrm{~S}$, or stage 4 aged $<18$ months at diagnosis (low and intermediate group) had a proportion of $2 \%$ for toxic death (maximum was $9 \%$ in trial NB79, minimum was $1 \%$ in trial NB2004). Within this group, chemotherapy was the cause of toxic death in $41 \%$ of cases, surgical complications in $38 \%$, and other events in $22 \%$. The decrease in the frequency of toxic death in the more recent trials is significant. Inclusion of the tumor/toxicity cases (discrimination of the causes "by toxicity" and/or "by tumor progression" impossible) adds $2 \%$ to the high-risk and $1 \%$ to the group with stages $1,2,3$, $4 \mathrm{~S}$, or 4 aged $<18$ months.

MYCN status has been available since 1990: the proportion of toxic deaths for high-risk patients was $4.4 \%$ ( $90 \%$ by chemotherapy, $9 \%$ by operation, $1 \%$ by other). Among children with normal MYCN status and clinically low and intermediate risks, the proportion of toxic deaths was $1 \%(22 / 2042 ; 54 \%$ by operations, $41 \%$ by chemotherapy, $5 \%$ by other causes).

\subsubsection{Time Intervals between Chemotherapy Cycles as a Measure of Toxicity}

The median time interval from day 1 of the first cycle to day 1 of the second chemotherapy cycle was 24 days (all 
Table 5 Frequencies of toxic death by trial

\begin{tabular}{|c|c|c|c|c|c|c|c|c|}
\hline & \multicolumn{4}{|c|}{ Stages $1-3 / 4 \mathrm{~S} / 4$ aged $<18 \mathrm{mo}$} & \multicolumn{4}{|c|}{ Stage 4 aged $\geq 18 \mathrm{mo}$} \\
\hline & \multicolumn{2}{|c|}{ By toxicity } & \multicolumn{2}{|c|}{ By toxicity and tumor } & \multicolumn{2}{|c|}{ By toxicity } & \multicolumn{2}{|c|}{ By toxicity and tumor } \\
\hline & $n / N$ & $\%$ & $n / N$ & $\%$ & $n / N$ & $\%$ & $n / N$ & $\%$ \\
\hline NB79 & $10 / 115$ & 8.8 & $1 / 115$ & 0.9 & $5 / 73$ & 6.8 & $2 / 73$ & 2.7 \\
\hline NB82 & $4 / 145$ & 2.8 & $5 / 145$ & 3.4 & $5 / 70$ & 7.1 & $3 / 70$ & 4.3 \\
\hline NB85 & $8 / 276$ & 2.9 & $2 / 276$ & 0.7 & $15 / 129$ & 11.6 & $1 / 129$ & 0.8 \\
\hline NB90 & $25 / 639$ & 3.9 & $6 / 639$ & 0.9 & $20 / 294$ & 6.8 & $7 / 294$ & 2.4 \\
\hline NB97 & $13 / 919$ & 1.4 & $3 / 919$ & 0.3 & $15 / 281$ & 5.3 & $5 / 281$ & 1.8 \\
\hline NB2004 & $12 / 1091$ & 1.1 & $4 / 1091$ & 0.4 & $16 / 425$ & 3.8 & $7 / 425$ & 1.6 \\
\hline All & $72 / 3185$ & 2.3 & $21 / 3181$ & 0.7 & $76 / 1272$ & 6.0 & $25 / 1272$ & 2.0 \\
\hline Cochrane-Armitage & \multicolumn{4}{|c|}{$P=0.010$} & \multicolumn{4}{|c|}{$P=0.001$} \\
\hline
\end{tabular}

trials). The time interval increased to 26 days from the second to the third cycle (day 1/day 1 ). The intervals were $21 / 23$ days in trial NB79, 23/23 days for NB82, 26/28 days for NB85, 28/28 days for NB90, 23/24 days for NB97, and 24/25 days for NB2004.

\subsubsection{Frequencies of Late Sequelae}

The most frequent late sequelae among all 3869 patients surviving $\geq 1$ year after diagnosis were renal dysfunctions (5.9\%), hypothyroidism (4.7\%), auditory impairment (4.1\%), spine deformities (3.1\%), second malignancies (2.0\%), other growth abnormalities, e.g., short stature $(1.8 \%)$, hepatic dysfunction (1.1\%), cardiologic impairment $(0.9 \%)$, and neurological sequelae $(0.4 \%)$.

Table 6 demonstrates the frequencies restricted to the group of patients with stage 4 disease aged $\geq 18$ months. The ranking order is similar but the frequency is significantly higher. Differences between the trials were noticed. Renal dysfunctions were most frequently observed in trial NB90 and decreased thereafter. In contrast, hypothyroidism and hearing impairment were increasingly detected. An exorbitant proportion of second malignancies $(8.9 \%)$ was observed in trial NB90, mainly in the maintenance chemotherapy arm. They were diagnosed up to 22 years after diagnosis. After closing that arm, the risk dropped back to $1-2 \%$ (maximum follow-up time was 19 years).

\section{Discussion}

The incidence of neuroblastoma has changed, the therapeutic intensity has been both reduced and intensified, and the survival probabilities have increased over recent decades in Germany. The strength of the present study is the 97\% relative frequency of participation in six consecutive national clinical trials of patients known to the central German Childhood Cancer Registry at the University of
Mainz. All trials were devoted to "neuroblastoma" in its entirety and not limited to selected sub-cohorts.

There are several limitations to this study. The number of patients with neuroblastoma who were not reported to the central German Childhood Cancer Registry is unknown and, although the collection quota after the late 1980s is assumed to exceed $95 \%$, this figure remains speculative. The frequency of participation in clinical trials was also not $100 \%$. Thus, the analyzed cohort may be considered representative but remains incomplete. Furthermore, the number of patients per year in Germany is statistically small, and the time period per trial and the related number of patients varied considerably. The diagnostic tools, e.g., the radiological imaging, histological, cytological, biochemical, and molecular techniques, improved significantly from 1979 to 2015 and, although key diagnostics were centrally reviewed, there were exceptions and not all patients had centrally reviewed procedures. Only three randomized controlled trials (RCTs) were conducted for high-risk patients during the analyzed period. Not all patients received the recommended treatment, and the grade of compliance was not evaluated (in order to analyze an unselected series of patients). No discrimination was made between "treatment per protocol" and "violation of protocol" (by drug dose, omission of drugs, use of other treatment elements, etc.). Finally, the developing skills of the medical professions ("learning curves") were not considered, e.g., the surgeon's understanding of useful aggressiveness, pediatric therapy of neutropenic febrile episodes, etc.

\subsection{The Change in Neuroblastoma Incidence}

The observed increase in neuroblastoma of $7 \%$ per 10 years, particularly for stages $1-3$ and stage $4 \mathrm{~S}$, does not truly indicate that more neuroblastomas have developed in recent times in Germany. The principal ability of low-risk tumors for spontaneous regression is well-known. The approximately twofold higher incidence rate of 
Table 6 Reported late effects in patients with stage 4 disease aged $>18$ months surviving $\geq 1$ year after diagnosis

\begin{tabular}{lccccccc}
\hline Trial & NB79 & NB82 & NB85 & NB90 & NB97 & NB04 & A1l \\
\hline$N$ & 46 & 47 & 91 & 235 & 250 & 364 & 1019 \\
Renal impairment & 0 & 2.1 & 4.4 & 19.6 & 13.2 & 4.7 & 9.9 \\
Thyroid dysfunction & 0 & 0 & 3.3 & 4.7 & 10 & 16.2 & 9.6 \\
Hearing loss & 0 & 0 & 1.1 & 3.8 & 8.4 & 10.2 & 6.7 \\
Second malignancy & 0 & 0 & 1.1 & 8.9 & 1.2 & 1.9 & 3.1 \\
Growth retardation & 0 & 4.3 & 2.2 & 4.7 & 2 & 1.9 & 2.6 \\
Hepatopathy & 0 & 0 & 0 & 3 & 1.6 & 4.1 & 2.6 \\
Spine deformaties & 0 & 0 & 2.2 & 4.7 & 1.6 & 0.8 & 2 \\
Cardiovascular dysfunction & 0 & 2.1 & 1.1 & 2.1 & 1.6 & 0.8 & 1.4 \\
Peripheral neuropathy & 0 & 0 & 0 & 0.4 & 0.8 & 0.5 & 0.5 \\
\hline
\end{tabular}

Data are presented as \%

a Second malignancies without time restrictions: any time after diagnosis (median time of observed cases 70 months, minimum 12 months, maximum 253 months) neuroblastomas in areas with neuroblastoma screening programs suggests that the undetected cases in areas without screening very likely developed but also regressed spontaneously [11]. After the end of the screening program in Germany, the incidence continued to be somewhat higher than before the screening. It is possible that a greater awareness and improved diagnostic facilities may play a role in this increase. Comparing incidence data from some sufficiently large and reliable registries (Australia, Canada, Netherlands, Norway, Switzerland, England, Wales, the Surveillance, Epidemiology, and End Results [SEER] program in the US White population) a general increase was observed except for Norway and Switzerland [16, 17]. The largest increases were seen in Germany and Canada, both of which had a screening program.

Significant differences in incidences between countries have been reported. The standardized incidence rates of neuroblastoma per 1 million population aged $0-14$ years varied between 3.8 (Mexico), 4.7 (Chile), 5.9 (Brazil), 8.3 (Argentina), 9.1 (Uruguay), 10.4 (USA), 10.9 (Europe), and 13.7 (Germany) [18]. An association between the global neuroblastoma incidence variations and socioeconomic status was assumed [19]. In non-metropolitan areas (USA), under-reporting and environmental factors were suspected as potential causes for the lower incidence [20]. For Germany, the cause for the increased incidence of lowrisk patients may be related to diagnostic awareness but remains unclear.

The increase in stage 4 disease among infants aged 0 to $<1$ year and the decrease in the group aged between 1 and $<2$ years may result from earlier diagnosis.

\subsection{The Change to Earlier Diagnoses}

The observed younger median age at diagnosis (decrease from 23 to 15 months for all stages) resulted mainly from earlier diagnosis of low- and intermediate-risk patients
(Table 2). If the stage 4 cohort was restricted to the highrisk group aged $\geq 18$ months, the median age at diagnosis remained at 41 months. The neuroblastoma screening project already demonstrated that the occurrence of highrisk neuroblastoma was not influenced by screening for neuroblastoma at 1 year [11]. This study adds that the age at presentation of the high-risk group remained unchanged.

The increase of the median age of stage $4 \mathrm{~S}$ infants by roughly 1 month may be explained by diagnostic restraint. The day of biopsy counted as the day of diagnosis, and biopsies were more delayed among the low-risk young infants. The widened diagnostic criteria for stage $4 \mathrm{~S}$ in Germany must be kept in mind and is reflected by, for example, the higher percentage of stage $4 \mathrm{~S}$ cases in Germany $(11 \%)$ compared with other countries such as Italy $(8 \%)$ [21].

The earlier diagnosis for all neuroblastoma must not be confused with the start of neuroblastoma growth in the patient.

\subsection{Disease Site Changes and Risk Factors}

Major changes in disease presentation and the composition of risk groups were not detected between the trials, with some exceptions. The number of unknown primaries decreased, which likely resulted from improved radiological imaging techniques. The decreasing incidence of liver metastasis (from 25 to $10 \%$ ) and the increasing detection of lung/pleura metastasis (from 3 to $6 \%$ ) cannot be explained. The proportions of abnormal LDH, ferritin, and chromosome $1 \mathrm{p}$ remained largely constant between the trials, with the exception of trial NB97 and its higher proportion of low-risk patients.

The cause of the MYCN amplification increase only in the stage 4 group aged $\geq 18$ months from $27 \%$ (NB97) to $35 \%$ (NB2004) is unclear. Different laboratory routines are unlikely, since the assignment to the categories "amplified 
$M Y C N$ " versus "non-amplified $M Y C N$ " required consistent results from two independent laboratories. The detection methods and the cut-offs were not changed. High throughput molecular data were not available for a clarifying comparative investigation.

\subsection{Surgery Alone for Low-Risk Neuroblastoma}

De Bernardi et al. [22] demonstrated that surgery alone is an effective and safe treatment for children with stage 1 (5-year EFS 94\%, 5-year OS 94\%, $n=288$ ) and stage 2 (5-year EFS $83 \%, 5$-year OS 93\%, $n=123$ ) neuroblastoma if the $M Y C N$ status is normal. The Children's Oncology Group [23] also reported excellent survival data for patients with stage 1 and 2 disease (5-year EFS 89\%, 5-year OS 97\%, $n=915$ ). Two to four cycles of chemotherapy were restricted to patients with organ- or life-threatening symptoms that could not be relieved by surgery and were applied in 115 children (13\%). There were no differences in EFS or OS between patients treated with surgery alone and those with immediate chemotherapy. The German NB97 trial treated infants with stage 1,2 , and 3 neuroblastoma with surgery alone if the patients had no organ- or life-threatening symptoms. The EFS differed significantly between resected and unresected tumors (3-year EFS for 190 patients with resected 85 vs. 56\% for 93 patients with unresected tumors), the OS proportions were identical (98 vs. 99\%) [24]. This means that recurrences were successfully treated with second-line surgery \pm chemotherapy. The proportion of patients with neuroblastoma who never received chemotherapy (at first line or recurrence) increased from $28 \%$ (NB85) to $60 \%$ (NB2004) for stages 1, 2, and 3 for all ages (Fig. 4). The feasibility of the "surgery alone concept" for stage 3 disease is supported by a retrospective institutional series of 14 patients treated by surgery only (A) in comparison with 39 patients who received pre-surgical chemotherapy with and without pre-surgical radiotherapy (B) [25]. The 10-year OS rates were $85 \%$ (A) and $97 \%$ (B) (log-rank $p=0.2$ ). More than one-third of those patients were aged $>24$ months (A: 5/14; B:16/39). Altogether, the discussed data support the usefulness of the "surgery alone" concept for stages 1,2, and 3 , provided there are no dangerous symptoms requiring immediate chemotherapy. The discrimination of stage 3 intermediate-risk from low-risk has not yet been solved. The currently used age cut-off needs to be confirmed or substituted, for example, by molecular profiling.

\subsection{Cyclic Chemotherapy for High-Risk Neuroblastoma}

The major cooperative groups considered carboplatin/cisplatin, cyclophosphamide/ifosfamide, doxorubicin, etoposide, and vincristine as key cytostatic drugs. They were used in various combinations with varying doses and application schedules and timing [26-30 and K. Matsumoto and M. Kumagai personal communication] (Table S5 in the ESM). The drugs were given in cycles lasting 3-8 days and required breaks of 2-3 weeks, mainly to recover from myelotoxicity. Dacarbazine, pirarubicine, topotecan, and vindesine were adopted by single groups for standard induction chemotherapy. Almost all drugs were selected by in vitro efficacy and/or by "clinical experience" [27], and their real impact is difficult to estimate. Randomized studies [29, 30] were the exceptions, which was due to the statistically low number of available patients. The increase in the EFS proportions obtained by all major study groups to a similar degree is surprising in view of the different doses, infusion times, and cycle structures. Researchers should keep in mind that the key group "stage 4 aged $\geq 18$ months" is rarely reported alone and that the specific contribution of various other high-risk groups (e.g., $M Y C N$-amplified localized neuroblastomas, stage 3 with unfavorable histology) are rarely highlighted. From over 3 decades of experience at the national and the international level, it appears unlikely that a re-design of cycles with known drugs alone will result in major steps forward.

\subsection{Myeloablative Therapy for High-Risk Neuroblastoma}

Three randomized trials on the use of myeloablative therapy with autologous stem cell transplantation have been published, and these demonstrated an advantage of myeloablative therapy in terms of EFS [31]. One major institution omitted the myeloablative regimen as a standard if consolidated with immunotherapy and isotretinoin [32]; others showed the feasibility and potential use of tandem transplants [34-35]. Tandem transplants are now the standard first-line therapy in the USA [33]. Busulfan/melphalan demonstrated a myeloablative regimen that is superior to melphalan/carboplatin/etoposide [36], but both combinations are still widely used (Table S5 in the ESM).

Haploidentical stem cell reconstitution together with interleukin-2 and antibody consolidation therapy [37] relies more on an immunotherapeutic concept than on eradication of residual tumor cells by myeloablative doses. This concept is still experimental.

\subsection{Surgical Therapy for High-Risk Neuroblastoma}

A systematic review including 13 studies on the use of aggressive surgical resection in stage 4 neuroblastoma [38] concluded that gross total resection resulted in a better 5 -year disease-free survival probability than subtotal resection (pooled odds ratio [OR] 1.55; 95\% CI 1.12-2.14; 
1665 patients). The 5-year OS probabilities were not improved (pooled OR 1.36; 95\% CI 0.96-1.91; 1778 patients). The series in our study was confined to patients with stage 4 aged $\geq 18$ months $(n=1116)$ and demonstrated superimposable EFS and OS curves up to 25 years between complete and incomplete resection (log-rank $p=0.270$ for EFS, $p=0.834$ for OS). In contrast, distinctly inferior EFS and OS were detected for the small group $(9.8 \%)$ with biopsies only. Since the best achieved surgical result per patient was used for this analysis (patients may have had several surgical procedures), the technical resectability, the aggressiveness of the pediatric surgeon, and the primary response to the preceding chemotherapy may all play a role. Furthermore, radiotherapy to the primary tumor may compensate for partial unresectability as shown for patients who still had active tumor residuals at the end of induction chemotherapy [39].

Overall, the use of technically difficult and long-lasting operations on high-risk primary tumors cannot be taken for granted.

\subsection{Long-Term Survival}

The 10-year EFS of the total cohort in this study improved from $44 \%$ (NB79) to 63\% (NB97), and the 10-year OS increased from $46 \%$ (NB79) to $75 \%$ (NB97). The 10-year survival proportions of the NB2004 study are lower $(55 \%$ for EFS, 72\% for OS). A detailed analysis is due in 2018. The Italian neuroblastoma group reported an increase in the 10-year OS from 35 to $65 \%$ in 2216 children diagnosed between 1979 and 2005 [21]. Moroz et al. [40] analyzed the data of 11,037 children who were reported to the International Neuroblastoma Risk Group (INRG) project from Australia, Europe (including the German data), Japan, and North America and described an increase in 5-year EFS from $43 \%$ during 1974-1989 to $68 \%$ during 1997-2002, and an increase in 5-year OS from 47 to $77 \%$. Thus, large cooperative groups observed a significant total increase of the survival proportions for patients with neuroblastoma.

While the low-risk neuroblastoma group showed an excellent outcome worldwide and only the treatment intensity varied, the survival of patients with high-risk neuroblastoma is still in the range of $30 \%$ after 10 years for the core group and must be regarded as more than unsatisfactory. Results are often difficult to compare since the "core group" (stage 4 aged $\geq 18$ months with and without $M Y C N$ amplification) is often diluted by other subgroups that are treated as high risk, such as stage 3 with unfavorable histology and $M Y C N$-amplified stages 1, 2, and 3. The North American Children's Oncology Group (COG) included stage 4 aged 1-18 years, stage 3 with unfavorable histology or abnormal ferritin, and stages $1-3$ and $4 \mathrm{~S}$ with amplification into the high-risk group. The 5-year OS among 3352 patients increased from 29\% (1990-1994) to $34 \%$ (1995-1999), to $47 \%$ (2000-2004), and to $50 \%$ (2005-2010) [41]. In 301 Japanese patients with stage 4 disease aged $>1$ year, the 5-year OS rates were 33-34\% for the subgroup with MYCN amplification (Germany 34\%) and $39-42 \%$ for the subgroup with normal MYCN [42] (Germany 47\%). The 10-year OS of 929 Italian children with stage 4 was 29\% [21] (Germany 28\%). Taking into account the various inclusion criteria, the outcome of highrisk neuroblastoma has been somewhat improved over the recent 3 decades without obvious major differences between the study groups. However, the levels achieved, of roughly $30 \%$ EFS and $35 \%$ OS after 10 years, remain disappointing. The curves continue to fall further up to 20 years (Tables S8 and S9 in the ESM, Fig. 5c, d).

Different results were obtained for the subgroups of infants with $M Y C N$-amplified metastatic neuroblastoma. Canete et al. [43] reported 24 patients with stage 4 disease and ten infants with stage $4 \mathrm{~S}$ disease. The OS after 2 years was $30 \%$ for both groups. The 5-year OS for German patients was $37 \%$ for stage 4 aged $<18$ months $(n=118)$ and $45 \%$ for stage $4 \mathrm{~S}(n=39)$ (Table S8 in the ESM). The differences cannot be explained, and the different definitions for infants for stage $4 \mathrm{~S}$ and stage 4 are unlikely to account for it.

In our opinion, the general implications of our study are (1) the presented data may be regarded as representative for neuroblastoma in general as well as for subgroups, although limitations exist; (2) the observed trends in the neuroblastoma incidence may be influenced by increased diagnostic awareness and by the phenomenon of spontaneous regression; (3) the reported improved survival of high-risk neuroblastoma is a global phenomenon.

The therapeutic approaches all observed the "maximum therapy approach," but differed in details (doses and timing of cyclic chemotherapy, use and aggressiveness of other therapeutic elements). The grade of improvement remains unsatisfactory and is associated with severe late sequelae for a substantial fraction of the long-term survivors.

Future research should focus on the following areas:

1. The implementation of new molecular patterns and characteristics. These promise to optimize the allocation of patients to the various risk groups, which are treated very differently, and to optimally identify potential targetable molecules and pathways right from the initial diagnosis and from relapse [44-46].

2. The realization of internationally coordinated, phase II-IV RCTs. The great potential of targeted therapies for neuroblastoma has been recently reviewed [47]. The pharmacological inhibition of the mutated or amplified anaplastic large-cell lymphoma kinase is a 
promising example in this field [48, 49]. Since the proportion of targetable molecules is so low [44], international collaboration is mandatory. The pivotal role of RCTs cannot be overemphasized and also applies for phase II, III, and IV trials. In retrospect, the paucity of RCTs during the 37-year period represents the most substantial shortcoming of the German neuroblastoma trial history.

3. The exploitation of the anti-neuroblastoma capacity of the patient's immune system. This field has gained much interest since the landmark study by $\mathrm{Yu}$ et al. [50], who used the anti-ganglioside GD2 antibody ch14.18, sargramostine (granulocyte macrophage-colony stimulating factor [GM-CSF]), interleukin-2, and isotretinoin. Other immunotherapeutic approaches with a focus on natural killer (NK) cells, T cells, chimeric antigen receptor $\mathrm{T}$ (CAR-T) cells, and macrophages have been recently developed $[37,51,52]$ but are still in preclinical or early clinical phases and need to demonstrate their potential usefulness.

\section{Conclusion}

The incidence of neuroblastoma has increased over the recent 3 decades in Germany, it is diagnosed earlier in life, and the risk factor profiles within the risk categories remain largely unchanged. The 10-year OS probabilities for all patients improved by $26 \%$. More than half of patients with stage 1-3 disease did not need chemotherapy at any time and had an excellent outcome. The survival probabilities of high-risk patients have improved since 1980, but they are still unsatisfactory, and these survivors show a high burden of late sequelae. This representative and unselected series of patients may provide a useful source for future studies in defined sub-cohorts.

\begin{abstract}
Acknowledgements The statistical support of Angela Ernst for this comprehensive analysis was extremely appreciated. This review would not have been possible without the outstanding ongoing dedicated work of many people, particularly those involved with the trial office (Barbara Hero, Thorsten Simon), the molecular investigations, the tissue asservation for the tumor bank (Matthias Fischer), the clinical work with the patients, the families, and the reporting by colleagues from up to 85 institutions. Last but not least, we acknowledge the indispensable cooperation of our patients, which was crucial for this review. Given this study's extensive timeline and the sheer number of people involved, we cannot possibly name everyone who contributed meaningfully, but their work is appreciated.
\end{abstract}

\section{Compliance with Ethical Standards}

Funding The clinical trials were funded by the German Ministry of Research and Technology (Grant DLR 01ZP8602/3), the German
Children's Cancer Aid (Deutsche Krebshilfe; Grants 70-443, 70-2290-Be I, T12/97/Be1, 70107712), the German Children's Cancer Foundation (Deutsche Kinderkrebsstiftung; Grants DKS 95.09; 2004.08; 2006.10), and the Children's Cancer Research Fund Neuroblastoma (Fördergesellschaft Kinderkrebs-Neuroblastom-Forschung; 3610062131).

Conflict of interest FB, CS, PK, and FL have no conflicts of interest.

Open Access This article is distributed under the terms of the Creative Commons Attribution-NonCommercial 4.0 International License (http://creativecommons.org/licenses/by-nc/4.0/), which permits any noncommercial use, distribution, and reproduction in any medium, provided you give appropriate credit to the original author(s) and the source, provide a link to the Creative Commons license, and indicate if changes were made.

\section{References}

1. Maris JM, Hogarty MD, Bagatell R, Cohn SL. Neuroblastoma. Lancet. 2007;369:2106-20.

2. Cheung NK, Dyer MA. Neuroblastoma: developmental biology, cancer genomics and immunotherapy. Nat Rev Cancer. 2013;13:397-411.

3. Maris JM. Recent advances in neuroblastoma. N Engl J Med. 2010;362:2202-11.

4. Louis CU, Shohet JM. Neuroblastoma: molecular pathogenesis and therapy. Ann Rev Med. 2015;66:49-63.

5. Kaatsch P, Grabow D, Spix C. German Childhood Cancer Registry-Annual Report 2016 (1980-2015). Institute of Medical Biostatistics, Epidemiology and Informatics (IMBEI) at the University Medical Center of the Johannes Gutenberg University Mainz, 2016. http://www.kinderkrebsregister.de. Accessed 20 June 2014.

6. Brodeur GM, Pritchard J, Berthold F, Carlsen NL, Castel V, Castelberry RP, De Bernardi B, Evans AE, Favrot M, Hedborg F, et al. Revisions of the international criteria for neuroblastoma diagnosis, staging, and response to treatment. J Clin Oncol. 1993;11:1466-77.

7. Berthold F, Trechow R, Utsch S, Zieschang J. Prognostic factors in metastatic neuroblastoma. A multivariate analysis of 182 cases. Am J Pediatr Hematol Oncol. 1992;14:207-15.

8. Spitz R, Hero B, Skowron M, Ernestus K, Berthold F. $M Y C N-$ status in neuroblastoma: Characteristics of tumours showing amplification, gain, and non-amplification. Eur $\mathrm{J}$ Cancer. 2004:40:2753-9.

9. Spitz R, Hero B, Ernestus K, Berthold F. FISH analyses for alterations in chromosomes 1, 2, 3, and 11 define high-risk groups in neuroblastoma. Med Pediatr Oncol. 2003;41:30-5.

10. Evans AE, D'Angio GJ, Randolph J. A proposed staging for children with neuroblastoma. Children's cancer study group A. Cancer. 1971;27:374-8.

11. Schilling FH, Spix C, Berthold F, Erttmann R, Fehse N, Hero B, Klein G, Sander J, Schwarz K, Treuner J, Zorn U, Michaelis J. Neuroblastoma screening at one year of age. N Engl J Med. 2002;346:1047-53.

12. European Organisation for Research and Treatment of Cancer. Common toxicity criteria (CTC) https://www.eortc.be/services/ doc/ctc/. Accessed 20 June 2014.

13. National Cancer Institute. Division of Cancer Treatment \& Diagnosis. Cancer Therapy Evaluation Program (CTEP). CTCAE criteria. https://ctep.cancer.gov/protocoldevelopment/.../docs/ ctcaev3.pdf. Accessed 20 June 2014. 
14. Brock PR, Bellman SC, Yeomans EC, Pinkerton CR, Pritchard J. Cisplatin ototoxicity in children: a practical grading system. Med Pediatr Oncol. 1991;19:295-300.

15. Royston P, Sauerbrei W. Multivariable model-building: a pragmatic approach to regression analysis based on fractional polynomials for modelling continuous variables. Chichester: Wiley; 2008.

16. Spix C, Pastore G, Sankila R, Stiller CA, Steliarova-Foucher E. Neuroblastoma incidence and survival in European children (1978-1997): report from the Automated Childhood Cancer Information System project. Eur J Cancer. 2006;42:2081-91.

17. Steliarova-Foucher E, Colombet M, Ries LAG, Hesseling P, Moreno F, Shin HY, Stiller CA. International Incidence of Childhood Cancer, volume III (electronic version). Lyon: International Agency for Research on Cancer; 2017. http://iicc.iarc.fr/ results/. Accessed 20 June 2014.

18. Moreno F, Lopez Marti J, Palladino M, Lobos P, Gualtieri A, Cacciavillano W. Childhood neuroblastoma: incidence and survival in Argentina. Report from the National Pediatric Cancer Registry, ROHA Network 2000-2012. Pediatr Blood Cancer. 2016;63:1362-7.

19. Kamihara J, Ma C, Fuentes Alabi SL, Garrido C, Frazier AL, Rodriguez-Galindo C, Orjuela MA. Socioeconomic status and global variations in the incidence of neuroblastoma: Call for support of population-based cancer registries in low-middle-income countries. Pediatr Blood Cancer. 2017;64:321-3.

20. Hsieh MH, Meng MV, Walsh TJ, Matthay KK, Baskin LS. Increasing incidence of neuroblastoma and potentially higher associated mortality of children from nonmetropolitan areas: analysis of the surveillance, epidemiology, and end results database. J Pediatr Hematol Oncol. 2009;3:942-6.

21. Haupt R, Garaventa A, Gambini C, Parodi S, Cangemi G, Casale F, Viscardi E, Bianchi M, Prete A, Jenkner A, Luksch R, Di Cataldo A, Favre C, D'Angelo P, Zanazzo GA, Arcamone G, Izzi GC, Gigliotti AR, Pastore G, De Bernardi B. Improved survival of children with neuroblastoma between 1979 and 2005: a report of the Italian Neuroblastoma Registry. J Clin Oncol. 2010;28:2331-8.

22. De Bernardi B, Mosseri V, Rubie H, Castel V, Foot A, Ladenstein R, Laureys G, Beck-Popovic M, de Lacerda AF, Pearson AD, De Kraker J, Ambros PF, de Rycke Y, Conte M, Bruzzi P, Michon J. Treatment of localised resectable neuroblastoma. Results of the LNESG1 study by the SIOP Europe Neuroblastoma Group. Br J Cancer. 2008;99:1027-33.

23. Strother DR, London WB, Schmidt ML, Brodeur GM, Shimada H, Thorner P, Collins MH, Tagge E, Adkins S, Reynolds CP, Murray K, Lavey RS, Matthay KK, Castleberry R, Maris JM, Cohn SL. Outcome after surgery alone or with restricted use of chemotherapy for patients with low-risk neuroblastoma: results of Children's Oncology Group study P9641. J Clin Oncol. 2012;30:1842-8.

24. Hero B, Simon T, Spitz R, Ernestus K, Gnekow AK, ScheelWalter HG, Schwabe D, Schilling FH, Benz-Bohm G, Berthold F. Localized infant neuroblastomas often show spontaneous regression: results of the prospective trials NB95-S and NB97. J Clin Oncol. 2008;26:1504-10.

25. Modak S, Kushner BH, LaQuaglia MP, Kramer K, Cheung NK. Management and outcome of stage 3 neuroblastoma. Eur J Cancer. 2009;45:90-8.

26. Park JR, Scott JR, Stewart CF, London WB, Naranjo A, Santana VM, Shaw PJ, Cohn SL, Matthay KK. Pilot induction regimen incorporating pharmacokinetically guided topotecan for treatment of newly diagnosed high-risk neuroblastoma: a Children's Oncology Group study. J Clin Oncol. 2011;29:4351-7.
27. Berthold F, Hero B. Neuroblastoma: current drug therapy recommendations as part of the total treatment approach. Drugs. 2000;59:1261-77.

28. Kushner BH, Kramer K, LaQuaglia MP, Modak S, Yataghene K, Cheung NK. Reduction from seven to five cycles of intensive induction chemotherapy in children with high-risk neuroblastoma. J Clin Oncol. 2004;22:4888-92.

29. Pearson AD, Pinkerton CR, Lewis IJ, Imeson J, Ellershaw C, Machin D, European Neuroblastoma Study Group; Children's Cancer and Leukaemia Group (CCLG formerly United Kingdom Children's Cancer Study Group). High-dose rapid and standard induction chemotherapy for patients aged over 1 year with stage 4 neuroblastoma: a randomised trial. Lancet Oncol. 2008;9:247-56.

30. London WB, Frantz CN, Campbell LA, Seeger RC, Brumback BA, Cohn SL, Matthay KK, Castleberry RP, Diller L. Phase II randomized comparison of topotecan plus cyclophosphamide versus topotecan alone in children with recurrent or refractory neuroblastoma: a Children's Oncology Group study. J Clin Oncol. 2010;28:3808-15.

31. Yalçin B, Kremer LC, van Dalen EC. High-dose chemotherapy and autologous haematopoietic stem cell rescue for children with high-risk neuroblastoma. Cochrane Database Syst Rev. 2015;(10):CD006301.

32. Kushner BH, Ostrovnaya I, Cheung IY, Kuk D, Modak S, Kramer K, Roberts SS, Basu EM, Yataghene K, Cheung NK. Lack of survival advantage with autologous stem-cell transplantation in high-risk neuroblastoma consolidated by anti-GD2 immunotherapy and isotretinoin. Oncotarget. 2016;7:4155-66.

33. Park JR, Kreissman SG, London WB, et al. A phase III randomized clinical trial (RCT) of tandem myeloablative autologous stem cell transplant (ASCT) using peripheral blood stem cell (PBSC) as consolidation therapy for high-risk neuroblastoma (HR-NB): a Children's Oncology Group (COG) study. J Clin Oncol. 2016;34 (Suppl):Abstract LBA3.

34. Granger M, Grupp SA, Kletzel M, Kretschmar C, Naranjo A, London WB, Diller L. Feasibility of a tandem autologous peripheral blood stem cell transplant regimen for high risk neuroblastoma in a cooperative group setting: a Pediatric Oncology Group Study. Pediatr Blood Cancer. 2012;59:902-7.

35. Pasqualini C, Dufour C, Goma G, Raquin MA, Lapierre V, Valteau-Couanet D. Tandem high-dose chemotherapy with thiotepa and busulfan-melphalan and autologous stem cell transplantation in very high-risk neuroblastoma patients. Bone Marrow Transplant. 2016;51:227-31.

36. Ladenstein R, Pötschger U, Pearson ADJ, Brock P, Luksch R, Castel V, Yaniv I, Papadakis V, Laureys G, Malis J, Balwierz W, Ruud E, Kogner P, Schroeder H, Forjaz de Lacerda A, BeckPopovic M, Bician P, Garami M, Trahair T, Canete A, Ambros PF, Holmes K, Gaze M, Schreier G, Garaventa A, Vassal G, Michon J, Valteau-Couanet D, For the SIOP Europe Neuroblastoma Group (SIOPEN). Busulfan and melphalan versus carboplatin, etoposide, and melphalan as high-dose chemotherapy for high-risk neuroblastoma (HR-NBL1/SIOPEN): an international, randomised, multi-arm, open- label, phase 3 trial. Lancet Oncol. 2017;18:500-14.

37. Kanold J, Paillard C, Tchirkov A, Lang P, Kelly A, Halle P, Isfan F, Merlin E, Marabelle A, Rochette E, Deméocq F. NK cell immunotherapy for high-risk neuroblastoma relapse after haploidentical HSCT. Pediatr Blood Cancer. 2012;59(4):739-42.

38. Mullassery D, Farrelly P, Losty PD. Does aggressive surgical resection improve survival in advanced stage 3 and 4 neuroblastoma? A systematic review and meta-analysis. Pediatr Hematol Oncol. 2014;31:703-16. 
39. Simon T, Hero B, Bongartz R, Schmidt M, Müller RP, Berthold F. Intensified external-beam radiation therapy improves the outcome of stage 4 neuroblastoma in children $>1$ year with residual local disease. Strahlenther Onkol. 2006;182:389-94.

40. Moroz V, Machin D, Faldum A, Hero B, Iehara T, Mosseri V, Ladenstein R, De Bernardi B, Rubie H, Berthold F, Matthay KK, Monclair T, Ambros PF, Pearson AD, Cohn SL, London WB. Changes over three decades in outcome and the prognostic influence of age-at-diagnosis in young patients with neuroblastoma: a report from the International Neuroblastoma Risk Group Project. Eur J Cancer. 2011;47:561-71.

41. Pinto NR, Applebaum MA, Volchenboum SL, Matthay KK, London WB, Ambros PF, Nakagawara A, Berthold F, Schleiermacher G, Park JR, Valteau-Couanet D, Pearson AD, Cohn SL. Advances in risk classification and treatment strategies for neuroblastoma. J Clin Oncol. 2015;33:3008-17.

42. Kaneko M, Tsuchida Y, Mugishima H, Ohnuma N, Yamamoto K, Kawa K, Iwafuchi M, Sawada T, Suita S. Intensified chemotherapy increases the survival rates in patients with stage 4 neuroblastoma with $M Y C N$ amplification. J Pediatr Hematol Oncol. 2002;24(8):613-21.

43. Canete A, Gerrard M, Rubie H, Castel V, Di Cataldo A, Munzer C, Ladenstein R, Brichard B, Bermúdez JD, Couturier J, de Bernardi B, Pearson AJ, Michon J. Poor survival for infants with MYCN-amplified metastatic neuroblastoma despite Intensified treatment: the International Society of Paediatric Oncology European Neuroblastoma Experience. J Clin Oncol. 2009;27:1014-9.

44. Schramm A, Köster J, Assenov Y, Althoff K, Peifer M, Mahlow E, Odersky A, Beisser D, Ernst C, Henssen AG, Stephan H, Schröder C, Heukamp L, Engesser A, Kahlert Y, Theissen J, Hero B, Roels F, Altmüller J, Nürnberg P, Astrahantseff K, Gloeckner C, De Preter K, Plass C, Lee S, Lode HN, Henrich KO, Gartlgruber M, Speleman F, Schmezer P, Westermann F, Rahmann S, Fischer M, Eggert A, Schulte JH. Mutational dynamics between primary and relapse neuroblastomas. Nat Genet. 2015;47:872-7.

45. Schleiermacher G, Janoueix-Lerosey I, Ribeiro A, Klijanienko J, Couturier J, Pierron G, Mosseri V, Valent A, Auger N, Plantaz D, Rubie H, Valteau-Couanet D, Bourdeaut F, Combaret V, Bergeron C, Michon J, Delattre O. Accumulation of segmental alterations determines progression in neuroblastoma. J Clin Oncol. 2010;28:3122-30.
46. Peifer M, Hertwig F, Roels F, Dreidax D, Gartlgruber M, Menon R, Krämer A, Roncaioli JL, Sand F, Heuckmann JM, Ikram F, Schmidt R, Ackermann S, Engesser A, Kahlert Y, Vogel W, Altmüller J, Nürnberg P, Thierry-Mieg J, Thierry-Mieg D, Mariappan A, Heynck S, Mariotti E, Henrich KO, Gloeckner C, Bosco G, Leuschner I, Schweiger MR, Savelyeva L, Watkins SC, Shao C, Bell E, Höfer T, Achter V, Lang U, Theissen J, Volland R, Saadati M, Eggert A, de Wilde B, Berthold F, Peng Z, Zhao C, Shi L, Ortmann M, Büttner R, Perner S, Hero B, Schramm A, Schulte JH, Herrmann C, O'Sullivan RJ, Westermann F, Thomas RK, Fischer M. Telomerase activation by genomic rearrangements in high-risk neuroblastoma. Nature. 2015;526:700-4.

47. Berlanga $P$, Cañete A, Castel V. Advances in emerging drugs for the treatment of neuroblastoma. Expert Opin Emerg Drugs. 2017;22:63-75.

48. Mossé YP, Lim MS, Voss SD, Wilner K, Ruffner K, Laliberte J, Rolland D, Balis FM, Maris JM, Weigel BJ, Ingle AM, Ahern C, Adamson PC, Blaney SM. Safety and activity of crizotinib for paediatric patients with refractory solid tumours or anaplastic large-cell lymphoma: a Children's Oncology Group phase 1 consortium study. Lancet Oncol. 2013;14:472-80.

49. Bresler SC, Weiser DA, Huwe PJ, Park JH, Krytska K, Ryles H, Laudenslager M, Rappaport EF, Wood AC, McGrady PW, Hogarty MD, London WB, Radhakrishnan R, Lemmon MA, Mossé YP. ALK mutations confer differential oncogenic activation and sensitivity to ALK inhibition therapy in neuroblastoma. Cancer Cell. 2014;26:682-94.

50. Yu AL, Gilman AL, Ozkaynak MF, London WB, Kreissman SG, Chen HX, Smith M, Anderson B, Villablanca JG, Matthay KK, Shimada H, Grupp SA, Seeger R, Reynolds CP, Buxton A, Reisfeld RA, Gillies SD, Cohn SL, Maris JM, Sondel PM, Children's Oncology Group. Anti-GD2 antibody with GM-CSF, interleukin-2, and isotretinoin for neuroblastoma. N Engl J Med. 2010;363:1324-34.

51. Mackall CL, Merchant MS, Fry TJ. Immune-based therapies for childhood cancer. Nat Rev Clin Oncol. 2014;11:693-703.

52. Borriello L, Seeger RC, Asgharzadeh S, DeClerck YA. More than the genes, the tumor microenvironment in neuroblastoma. Cancer Lett. 2016;380:304-14. 